\title{
Small animal imaging presents an opportunity for improving translational research in biological psychiatry
}

\author{
M. Mallar Chakravarty, PhD; Elisa Guma, PhD
}

Recent efforts in biological psychiatry have made a significant push toward examining both psychopathology and brain circuitry on a continuum across the spectrum of healthy individuals to those suffering from chronic and unremitting neuropsychiatric disorders. ${ }^{1}$ Neuroimaging methods have provided a critical tool through which to examine this continuum, ${ }^{2,3}$ given their noninvasive, repeatable nature. Additionally, they provide a scaffold through which one can relate sources of neurobiological heterogeneity (in the form of brain anatomy and function) to clinical and behavioural heterogeneity. ${ }^{4-6}$ In particular, magnetic resonance imaging (MRI) methods have been important in this regard, given the public availability of data and the multiple analysis streams that leverage different tissue properties to make inferences on brain topology and circuitry. ${ }^{78}$ Arguably, this sustained strategy contributed to recent significant advances in using knowledge of brain circuitry to target novel brain stimulation approaches as treatments for neuropsychiatric disorders ${ }^{9,10}$ and for improving our understanding of the mechanism of action of pharmacological agents (like ketamine) being repurposed for use in different clinical contexts. ${ }^{11}$ In aggregate, these developments are highly suggestive of how significant gains in novel therapeutics can be obtained using reliable investigations of well-characterized brain circuits.

Despite these important advancements, there is still much work to do in understanding how neuropsychiatric disorders emerge over time. Human neuroimaging experiments, MRI in particular, lack the important ability to achieve detailed mechanistic insights that involve cellular- or molecular-level information. There are notable exceptions, such as the use of positron emission tomography or magnetic resonance spectroscopy (and related techniques), for obtaining data on specific molecular phenotypes. ${ }^{12,13}$ Alternatively, one can relate neural phenotypes ${ }^{14-16}$ to gene expression data from public resources like the Allen Human Brain Atlas; ${ }^{17}$ however, these relationships are generally derived neuroinformatically and preclude the investigation of cellular and molecular pheno- types. There are limited means to perform detailed investigations across architectural scales (molecular, cellular, structural and functional connectivity, and morphology) in humans as one could perform using animal models of the central nervous system disorders. To this end, small animal imaging may provide meaningful opportunities in the context of biological psychiatry for bridging between macro- and microanatomic scales of brain circuitry. In this editorial, we describe both the utility of MRI research (with an emphasis on the use of rodent models) for gaining mechanistic insight into neuropsychiatric illness and how dimensional approaches that have provided important advances in the clinical/neuroimaging literature can be translated into experimental design.

\section{Opportunities to use small animal imaging for discovery in biological psychiatry}

Small animal neuroimaging is one of the few techniques in neuroscience where investigations in human and clinical populations preceded the detailed work that is beginning to emerge in experimental models. Thus, there remains a methodological barrier to the initiation of experiments using small animals. While there are important logistical and technical challenges that must be overcome in order to achieve a reliable signal that can be used for analyses, ${ }^{18-21}$ these will not be addressed in this editorial; however, we suggest designing experiments with these challenges in mind. Nonetheless, there are considerable advantages to using small-animal neuroimaging that include the controlled experimental manipulations that can be done both in and out of the scanner; postmortem assessments for achieving translational insight; and the relatively short gestational, developmental, and aging periods that are ideal for longitudinal experiments.

In its infancy, the field of small animal neuroimaging leveraged MRI-based techniques to examine the structural and functional phenotypes related to genetically modified animal

Correspondence to: M.M. Chakravarty and E. Guma, Douglas Hospital Research Institute, 6875 Blvd LaSalle, Verdun QC H4H 1R3; mallar@cobralab.ca and elisa.guma@mail.mcgill.ca

Cite as: J Psychiatry Neurosci 2021 October 20;46(5). doi: 10.1503/jpn.210172 
models ${ }^{22-24}$ or environmental exposures ${ }^{25}$ typically in crosssectional settings. This type of study design provides an important and useful starting point in the investigation of altered brain circuitry; however, it only scratches the surface of opportunities that animal MRI provides. One of the challenges in the use of experimental models is appropriately capturing and investigating the heterogeneity that is typically observed in neuropsychiatric diagnoses. This is especially important, given the dynamic nature of brain changes in response to environmental risk factors and treatments ${ }^{5}$ and the neurodevelopmental underpinnings of many disorders, ${ }^{26,27}$ which likely contribute to the variability observed ${ }^{3}$ (e.g., several risk genotypes may map onto a single clinical diagnosis, or vice versa). This variability may also be a source of important information and potentially a key feature that needs to be better understood in order to make advances in our understanding of neuropsychiatric illness. In many animal models of neurodevelopmental or psychiatric disorders, the differences in effect sizes of treatment outcomes between experimental and control groups can be difficult to ascertain. However, leveraging this variability by harnessing clustering techniques from data science ${ }^{28}$ or examining susceptibility or resilience ${ }^{29}$ to a specific environmental risk factor (e.g., maternal immune activation [MIA $]^{30}$ ) are important strategies that allow for a more nuanced understanding of the spectrum of phenotypes. While these approaches are critical to examining the heterogeneous nature of the model organisms and, ultimately, the disorders experimenters are seeking to model, they still segregate or use specific, discrete categorizations rather than examine a dimension of brain and behaviour phenotypes. Despite these issues of intersubject variability, the availability of rodent models in biological psychiatry research has led to useful and important discoveries, such as identifying therapeutic strategies related to antidepressant action $^{31}$ (among other key findings).

Recent work from our group has provided a window into how this dimensional approach could be achieved. We examined multiple behaviours across different developmental stages in the same animal and demonstrated how they may be related to brain development using longitudinal structural neuroimaging in MIA-exposed mice. ${ }^{32}$ Rather than using a single behavioural phenotype, we chose to use partial least squares $^{33}$ analysis that generated, in a data-driven fashion, linked dimensions of MIA-exposure-related variation in behaviour and brain development, where each mouse could be examined within a space of latent variables derived from within the data set. This analysis approach has proven successful in the human neuroimaging literature and provides an example of successful translation across species using comparable modalities. In order to harness this variability to the benefit of the scientific investigations, researchers may need to use new methodological approaches and statistical analyses that allow for the identification of differences along dimensions rather than discrete categories. This strategy could be augmented further by using other more "naturalistic" behaviours, such as variability in spontaneous home cage activity, ${ }^{34}$ complementing the more standard tests we used previously.
Furthermore, in order to fully appreciate these subtle differences within the models related to neuropsychiatric disorders, it is critical to take a more integrative approach. ${ }^{32}$ Some groups have started to do this; for example, recent work by Mueller and colleagues ${ }^{29}$ used clustering approaches on a large sample of behaviourally phenotyped MIA offspring and identified subgroups of MIA offspring that were more or less behaviourally impaired. These subgroups were further found to differ based on transcriptional profiles and structural covariance, possibly linked to differences in inflammatory cytokine profiles. ${ }^{29}$ Similarly, we have demonstrated ${ }^{32}$ that using the linked brain-behaviour dimensions that we described above, we can identify transcriptional profiles related to autistic behaviours, inflammatory pathways and microRNA regulation. These critical integrative approaches start parsing the large set of biological factors that may be at play for any neuropsychiatric disorder. We believe that neuroimaging approaches may provide a useful scaffold through which to link biological data across different systems and varying scales of resolution.

\section{Considering rodent models in a neurodevelopmental context using neuroimaging}

In addition to the possibility of using multi-dimensional techniques, MRI-based studies of rodent models allow for the unique ability to investigate neural phenotypes at the wholebrain level and longitudinally. Given that the onset of many neuropsychiatric disorders occurs in childhood and adolescence, ${ }^{26,27}$ it is imperative that studies of rodent models aimed at investigating the neurobiology of these disorders leverage the unique ability of MRI to generate spatiotemporal maps of brain variation. Recent important work has gone into leveraging the availability of longitudinal methodologies in rodent models ${ }^{32,35-37}$ in both acquisition and statistical analysis. This type of work provides an important advance in our ability to characterize animal models and provides homology to human longitudinal neuroimaging studies that seek to examine neuropsychiatric disorders as deviations from normative developmental processes - a strategy that has provided critical insight into a range of neuropsychiatric disorders with neurodevelopmental underpinnings. ${ }^{38-40}$ However, special care should be taken to assess and carefully understand limitations that may be caused by repeat assessments. Previous studies that examined the impact of repeated imaging and anesthesia found limited effects on behavioural and neuroanatomical phenotypes, but more work would be useful to examine these confounds. ${ }^{35}$

Using animal models has many advantages and is an essential tool for testing causality and identifying molecular mechanisms. ${ }^{41}$ However, the utility of this strategy needs to be considered in the context of a few limitations. The use of any animal model to study human neuropsychiatric disorders or associated risk factors is extremely challenging because of the subjective nature of many core symptoms currently used to diagnose the disorders. To further complicate the problem, there are no objective biomarkers that 
map onto specific symptoms used for diagnosis. ${ }^{42}$ Therefore, examining risk factors or genotypes is critical to future studies using neuroimaging and small-animal models. Nonetheless, there are considerable benefits conferred by longitudinal MRI, such as whole-brain assays that may provide insight on where one should and could investigate further. While MRI lacks specificity to individual molecular and cellular mechanisms, ${ }^{43}$ it is a critical spotlight that can help integrate brain structure and function in the context of biological psychiatry.

Taken together, we offer the following recommendations as a means of developing methods that can help improve our understanding of mechanistic and phenotypic signatures related to neuropsychiatric disorders. Chiefly, we suggest that improving longitudinal methodologies such that they integrate more translationally relevant behaviour (e.g., behavioural tests performed in humans and adapted to work in touchscreens for rodents ${ }^{44}$ ) is critical to creating more robust models of neuropsychiatric disorders. We further suggest that integration of transcriptomics, neurochemical and deeper cellular-level phenotypes (derived using advanced microscopy techniques) may allow individual-level phenotyping that may provide a more nuanced and detailed understanding of genetics, environmental risk factors and heterogeneity in treatment response. This will, in turn, require the back-propagation of advanced data science and machine learning techniques ${ }^{2,3,30}$ that may enable the ability to make sense of "big data" at the level of the individual subject.

Affiliations: From the Computational Brain Anatomy (CoBrA) Laboratory, Cerebral Imaging Centre, Douglas Research Institute, Montreal, Que., Canada (Chakravarty, Guma); the Department of Psychiatry, McGill University, Montreal, Que., Canada (Chakravarty); the Department of Biological and Biomedical Engineering, McGill University, Montreal, Que., Canada (Chakravarty); and the Developmental Neurogenomics Unit, Human Genetics Branch, National Institute of Mental Health, Intramural Program, USA (Guma).

Competing interests: E. Guma declares trainee funding from Fonds du Recherche du Quebec en Sante. No other competing interests declared.

Content licence: This is an Open Access article distributed in accordance with the terms of the Creative Commons Attribution (CC BY-NC-ND 4.0) licence, which permits use, distribution and reproduction in any medium, provided that the original publication is properly cited, the use is noncommercial (i.e., research or educational use), and no modifications or adaptations are made. See: https: / / creativecommons.org/licenses/by-nc-nd/4.0/

\section{References}

1. Insel T, Cuthbert B, Garvey M, et al. Research domain criteria (RDoC): toward a new classification framework for research on mental disorders. Am J Psychiatry 2010;167:748-51.

2. Xia CH, Ma Z, Ciric R, et al. Linked dimensions of psychopathology and connectivity in functional brain networks. Nat Commun 2018;9:3003.

3. Park MTM, Raznahan A, Shaw P, et al. Neuroanatomical phenotypes in mental illness: identifying convergent and divergent cortical phenotypes across autism, ADHD and schizophrenia. J Psychiatry Neurosci 2018;43:201-12.

4. Olafson E, Bedford SA, Devenyi GA, et al. Examining the boundary sharpness coefficient as an index of cortical microstructure in autism spectrum disorder. Cereb Cortex 2021;31:3338-52.
5. Bedford SA, Park MTM, Devenyi GA, et al. Large-scale analyses of the relationship between sex, age and intelligence quotient heterogeneity and cortical morphometry in autism spectrum disorder. Mol Psychiatry 2020;25:614-28.

6. Moreau C, Urchs S, Huguet G, et al. Functional connectivity analyses suggest shared molecular mechanisms across 12 neuropsychiatric mutations, autism and schizophrenia. Biol Psychiatry 2020;87:S395.

7. Lerch JP, van der Kouwe AJW, Raznahan A, et al. Studying neuroanatomy using MRI. Nat Neurosci 2017;20:314-26.

8. Bullmore E, Sporns O. Complex brain networks: graph theoretical analysis of structural and functional systems. Nat Rev Neurosci 2009;10: 186-98.

9. Boes $\mathrm{AD}$, Uitermarkt BD, Albazron FM, et al. Rostral anterior cingulate cortex is a structural correlate of repetitive TMS treatment response in depression. Brain Stimul 2018;11:575-81.

10. Fox MD, Buckner RL, Liu H, et al. Resting-state networks link invasive and noninvasive brain stimulation across diverse psychiatric and neurological diseases. Proc Natl Acad Sci U S A 2014;111:E4367-75.

11. Sahib AK, Loureiro JR, Vasavada M, et al. Modulation of the functional connectome in major depressive disorder by ketamine therapy. Psychol Med 2020;doi: 10.1017/S0033291720004560.

12. Hafizi S, Guma E, Koppel A, et al. TSPO expression and brain structure in the psychosis spectrum. Brain Behav Immun 2018;74:79-85.

13. Suridjan I, Rusjan P, Addington J, et al. Dopamine D2 and D3 binding in people at clinical high risk for schizophrenia, antipsychotic-naive patients and healthy controls while performing a cognitive task. J Psychiatry Neurosci 2013;38:98-106.

14. Grasby KL, Jahanshad N, Painter JN, et al. The genetic architecture of the human cerebral cortex. Science 2020;367:eaay6690.

15. Reardon PK, Seidlitz J, Vandekar S, et al. Normative brain size variation and brain shape diversity in humans. Science 2018;360: 1222-27.

16. Richiardi, J, Altmann, A, Milazzo, AC, et al. Correlated gene expression supports synchronous activity in brain networks. Science 2015;348:1241-44.

17. Hawrylycz MJ, Lein ES, Guillozet-Bongaarts AL. An anatomically comprehensive atlas of the adult human brain transcriptome. Nature 2012;489:391-99.

18. Nieman BJ, Bock NA, Bishop J, et al. Magnetic resonance imaging for detection and analysis of mouse phenotypes. NMR Biomed 2005;18:447-68.

19. Grandjean J, Canella C, Anckaerts C, et al. Common functional networks in the mouse brain revealed by multi-centre resting-state fMRI analysis. Neuroimage 2020;205:116278.

20. Mandino F, Cerri DH, Garin CM, et al. Animal functional magnetic resonance imaging: trends and path toward standardization. Front Neuroinform 2020;13:78.

21. Nieman BJ, Bishop J, Dazai J, et al. MR technology for biological studies in mice. NMR Biomed 2007;20:291-303.

22. Johnson GA, Ali-Sharief A, Badea A et al. High-throughput morphologic phenotyping of the mouse brain with magnetic resonance histology. Neuroimage 2007;37:82-9.

23. Lerch JP, Carroll JB, Spring S, et al. Automated deformation analysis in the YAC128 Huntington disease mouse model. Neuroimage 2008;39:32-9.

24. Grandjean J, Schroeter A, He P, et al. Early alterations in functional connectivity and white matter structure in a transgenic mouse model of cerebral amyloidosis. J Neurosci 2014;34:13780-9.

25. Lerch JP, Yiu AP, Martinez-Canabal A, et al. Maze training in mice induces MRI-detectable brain shape changes specific to the type of learning. Neuroimage 2011;54:2086-95.

26. Keshavan MS, Paus T. Neurodevelopmental trajectories, disconnection, and schizophrenia risk. JAMA Psychiatry 2015;72:943-5.

27. Paus T, Keshavan M, Giedd JN. Why do many psychiatric disorders emerge during adolescence? Nat Rev Neurosci 2008;9:947-57. 
28. Ellegood J, Anagnostou E, Babineau BA, et al. Clustering autism: using neuroanatomical differences in 26 mouse models to gain insight into the heterogeneity. Mol Psychiatry 2015;20:118-25.

29. Mueller FS, Scarborough J, Schalbetter SM, et al. Behavioral, neuroanatomical, and molecular correlates of resilience and susceptibility to maternal immune activation. Mol Psychiatry 2021;26:396-410.

30. Guma E, Plitman E, Chakravarty MM. The role of maternal immune activation in altering the neurodevelopmental trajectories of offspring: a translational review of neuroimaging studies with implications for autism spectrum disorder and schizophrenia. Neurosci Biobehav Rev 2019;104:141-57.

31. Ramaker MJ, Dulawa SC. Identifying fast-onset antidepressants using rodent models. Mol Psychiatry 2017;22:656-65.

32. Guma E, do Couto Bordignon P, Devenyi GA, et al. Early or late gestational exposure to maternal immune activation alters neurodevelopmental trajectories in mice: an integrated neuroimaging, behavioural, and transcriptional study. Biol Psychiatry 2021;90:328-41.

33. McIntosh AR, Bookstein FL, Haxby JV, et al. Spatial pattern analysis of functional brain images using partial least squares. Neuroimage 1996;3:143-57.

34. Kobayashi K, Ikeda Y, Suzuki H. Behavioral destabilization induced by the selective serotonin reuptake inhibitor fluoxetine. Mol Brain 2011;4:12.

35. Qiu LR, Fernandes DJ, Szulc-Lerch KU, et al. Mouse MRI shows brain areas relatively larger in males emerge before those larger in females. Nat Commun 2018:9:2615.
36. Guma E, Rocchetti J, Devenyi GA, et al. Regional brain volume changes following chronic antipsychotic administration are mediated by the dopamine D2 receptor. Neuroimage 2018;176:226-38.

37. Kong V, Devenyi GA, Gallino D, et al. Early-in-life neuroanatomical and behavioural trajectories in a triple transgenic model of Alzheimer's disease. Brain Struct Funct 2018;223:3365-82.

38. Raznahan A, Shaw P, Lalonde F, et al. How does your cortex grow? J Neurosci 2011;31:7174-7.

39. Shaw P, Sharp W, Sudre G, et al. Subcortical and cortical morphological anomalies as an endophenotype in obsessive-compulsive disorder. Mol Psychiatry 2015;20:224-31.

40. Chakravarty MM, Rapoport JL, Giedd JN, et al. Striatal shape abnormalities as novel neurodevelopmental endophenotypes in schizophrenia: a longitudinal study. Hum Brain Mapp 2015;36: 1458-69.

41. Kentner AC, Bilbo SD, Brown AS, et al. Maternal immune activation: reporting guidelines to improve the rigor, reproducibility, and transparency of the model. Neuropsychopharmacology 2019;44:245-58.

42. Nestler EJ, Hyman SE. Animal models of neuropsychiatric disorders. Nat Neurosci 2010;13:1161-9.

43. Tardif CL, Gauthier CJ, Steele CJ, et al. Advanced MRI techniques to improve our understanding of experience-induced neuroplasticity. Neuroimage 2016;131:55-72.

44. Mar AC, Horner AE, Nilsson SRO, et al. The touchscreen operant platform for assessing executive function in rats and mice. Nat Protoc 2013;8:1985-2005. 